\title{
Stimulated Raman gain scattering in thin planar dielectric waveguides: erratum
}

Johannes Sake Kanger, Cees Otto, and Jan Greve

Applied Optics Group, Department of Applied Physics, BMTI and MESA Institutes,

University of Twente, P.O. Box 217, 7500 AE Enscheda, The Netherlands

In our recent Letter ${ }^{1}$ a numerical error was introduced in the calculation of the gain $G_{0}$. The values of the gain $G_{0}$ given in Figs. 2 and 3 should be multiplied by a factor of 5 to yield the correct values. Since this results in a gain that is even larger than that given in the Letter, it by no means alters the discussed ideas and conclusions.

\section{Reference}

1. J. S. Kanger, C. Otto, and J. Greve, Opt. Lett. 20, 2231 (1995). 